\title{
Purifications and Characterizations of a Ferredoxin and Its Related 2-Oxoacid:Ferredoxin Oxidoreductase from the Hyperthermophilic Archaeon, Sulfolobus solfataricus P1
}

\author{
Young-Jun Park, Chul-Bae Yoo, Soo Young Choi ${ }^{1}$ and Hee-Bong Lee* \\ Department of Biochemistry, College of Natural Sciences, Kangwon National University, Chuncheon, 200-701, Korea \\ ${ }^{1}$ Department of Biomedical Sciences and Institute for Bioscience and Biotechnology, Hallym University, Chuncheon, 200-702, Korea
}

Received 14 September 2005, Accepted 7 October 2005

\begin{abstract}
The coenzyme A-acylating 2-oxoacid:ferredoxin oxidoreductase and ferredoxin (an effective electron acceptor) were purified from the hyperthermophilic archaeon, Sulfolobus solfataricus P1 (DSM1616). The purified ferredoxin is a monomeric protein with an apparent molecular mass of approximately $11 \mathrm{kDa}$ by SDS-PAGE and of 11,180 \pm 50 Da by MALDI-TOF mass spectrometry. Ferredoxin was identified to be a dicluster, [3Fe-4S][4Fe-4S], type ferredoxin by spectrophotometric and EPR studies, and appeared to be zinc-containing based on the shared homology of its $\mathrm{N}$ terminal sequence with those of known zinc-containing ferredoxins. On the other hand, the purified 2-oxoacid: ferredoxin oxidoreductase was found to be a heterodimeric enzyme consisting of $69 \mathrm{kDa} \alpha$ and $34 \mathrm{kDa} \beta$ subunits by SDS-PAGE and MALDI-TOF mass spectrometry. The purified enzyme showed a specific activity of 52.6 units/mg for the reduction of cytochrome $c$ with 2-oxoglutarate as substrate at $5^{\circ} \mathrm{C}, \mathrm{pH} 7.0$. Maximum activity was observed at $70^{\circ} \mathrm{C}$ and the optimum $\mathrm{pH}$ for enzymatic activity was 7.0 -8.0. The enzyme displays broad substrate specificity toward 2-oxoacids, such as pyruvate, 2-oxobutyrate, and 2-oxoglutarate. Among the 2-oxoacids tested (pyruvate, 2oxobutyrate, and 2-oxoglutarate), 2-oxoglutarate was found to be the best substrate with $K_{m}$ and $\boldsymbol{k}_{\text {cat }}$ values of $163 \mu \mathrm{M}$ and $452 \mathrm{~min}^{-1}$, respectively. These results provide useful information for structural studies on these two proteins and for studies on the mechanism of electron transfer between the two.
\end{abstract}

Keywords: Archaea, Ferredoxin, 2-Oxoacids:ferredoxin oxidoreductase, Sulfolobus solfataricus, Thermostability

\footnotetext{
*To whom correspondence should be addressed.

Tel: 82-33-250-8512; Fax: 82-33-242-0459

E-mail: leehbong@kangwon.ac.kr
}

\section{Introduction}

Ferredoxins are present in all organisms, from bacteria and archaea to higher eukaryotes. They are small and acidic ironsulfur proteins with prosthetic groups, composed of iron and sulfur atoms, which are referred to as iron-sulfur clusters. The ferredoxins function as intracellular electron carriers with a low reduction potential (Mortenson et al., 1962; Beinert, 1990; Johnson, 1994). In addition to their roles in electron transfer reactions, iron-sulfur clusters are also now known to participate in the activation of substrates (Beinert et al., 1996; Broderick, et al., 1997), the stabilization of radicals and structures (Howard and Rees, 1996), the protection of proteins from enzymes (Grandoni et al., 1989), and the storage of iron and sulfur (Thauer and Schonheit, 1982), in addition, they act as sensors of iron, dioxygen, the superoxide ion $\left(\mathrm{O}_{2}^{-}\right)$, and possibly nitric oxide (Hentze and Kunh, 1996), and participate in gene expression (Michaels et al., 1990).

Ferredoxins are classified into three groups according to the types and numbers of their Fe-S clusters. The first group consists of monomeric (ca. 90-130 residues) proteins containing one $[2 \mathrm{Fe}-2 \mathrm{~S}]$ cluster, which includes ferredoxins that transfer electrons in redox chains linked to hydroxylases and oxygenases in a wide range of organisms (from bacteria to mammals) (Grinberg et al., 2000), and which includes plant and algal ferredoxins that function as electron carriers in photosynthesis (Matsubara and Sacki, 1992). The other two groups consist of ubiquitous proteins containing [3Fe-4S]/ [4Fe-4S] and are typically found in bacteria. They may differ in cluster type ( $3 \mathrm{Fe}$ or $4 \mathrm{Fe}$ ), number (one or two), and length of polypeptide chain (up to over 100 residues), and include the relatively small (ca. 55 residues) $2[4 \mathrm{Fe}-4 \mathrm{~S}]$ ferredoxins that were first isolated from anaerobic bacteria (Mortenson et al., 1962).

Ferredoxins are widely distributed in archaea, and occur in methanogens, halophiles, and thermophiles (Kerscher and Oesterhelt, 1981; Daas et al., 1994; Breton et al., 1995). To 
date the major types of clusters found in archaeal ferredoxins are as follows: [2Fe-2S] cluster found in a ferredoxin of Sulfolobus solfataricus (Kounosu et al., 2004), [4Fe-4S] cluster in a ferredoxin of Pyrococcus furiosus (Aono et al., 1989), and [3Fe-4S] [4Fe-4S] di-clusters in a ferredoxin of Acidianus ambivalens (Gomes et al., 1998), Desulfurolobus ambivalens (Teixeira et al., 1995), Sulfolobus acidocaldarius (Breton et al., 1995), Sulfolobus sp. strain 7 (Iwasaki et al., 1994), and Thermoplasama acidophilum (Iwasaki et al., 1997).

Although most thermophilic archaea are anaerobic organisms due to the scarcity of oxygen in their environments, one of the characteristic features in the central metabolic pathways of aerobic and anaerobic archaea is that ferredoxin functions as a physiological electron acceptor of the archaeal coenzyme Aacylating 2-oxoacid:ferredoxin oxidoreductase (Kerscher et al., 1982). Coenzyme A-acylating 2-oxoacid:ferredoxin oxidoreductases (100-300 kDa) in archaea (Kerscher and Oesterhelt, 1981; Blamey and Adams, 1993; Kunow et al., 1995; Zhang et al., 1996) play central roles in the decarboxylation of pyruvate and 2-oxoglutarate, rather than the $\mathrm{NAD}^{+}$-dependent 2oxoacid dehydrogenase multienzyme complexes $(2,000-7,000$ $\mathrm{kDa}$ ) which play similar roles in bacteria and eukaryotic mitochondria (Koike and Koike, 1976), and they contain one TPP and at least one [4Fe-4S] cluster (per protomer) as prosthetic groups (Zhang et al., 1996). The cofactor composition of archaeal enzymes is similar to that of enzymes from fermentative and phototrophic obligatory anaerobic bacteria (Meinecke et al., 1989), several photosynthetic bacteria (Brostedt and Nordlund, 1991), and anaerobic and amitochondrial protozoa (Williams et al., 1987). The 2-oxoacid:ferredoxin oxidoreductase from Sulfolobus sp. strain 7 is known to consist of $70-\mathrm{kDa} \alpha$ and $37-\mathrm{kDa} \beta$ subunits, and to contain one TPP and one [4Fe-4S] cluster, and two Mg atoms per $\alpha \beta$ structure (Zhang et al., 1996).

S. solfataricus P1 (DSM1616) belongs to the thermoacidophilic archaea, which inhabit sulfur-rich volcanic hot springs, a high temperature/low $\mathrm{pH}$ environment (Brock et al., 1972; Rosa et al., 1975; Zillig et al., 1980). In this paper, we report for the first time on the purification and characterization of thermostable ferredoxin and 2-oxoacid:ferredoxin oxidoreductase from $S$. solfataricus P1 strain. The purified ferredoxin was identified as an electron carrier containing dicluster, [3Fe-4S] [4Fe-4s], and the purified 2-oxoacid:ferredoxin oxidoreductase was found to be a heterodimeric enzyme, which showed a fastest turnover rate of 2-oxoglutarate than of the two other oxoacids, pyruvate and 2-oxobutyrate.

\section{Materials and Methods}

Materials. Sulfolobus solfataricus P1 (DSM1616) was purchased from the American Type Culture Collection. Yeast extract was purchased from Difco Laboratories and DEAE-Sephacel, Phenyl Sepharose CL-4B, Q-Sepharose Fast Flow, Superdex 200, Sephadex G-50, and Blue Sepharose 6 fast flow were from Pharmacia Biotech. 2Oxoglutarate and pyruvate were purchased from Biochemical. All other chemicals used were purchased from Sigma (St. Louis) unless otherwise stated.

Organism and growth conditions. S. solfataricus P1 (DSM1616) was aerobically cultivated at $75^{\circ} \mathrm{C} / \mathrm{pH} 4.0$ in a $5 \mathrm{~L}$ fermentor with moderate stirring $(120 \mathrm{rpm})$. The medium containing $0.22 \%(\mathrm{w} / \mathrm{v})$ yeast extract and the mineral bases was as described for ATCC media formulation (Zillig et al., 1980). Cells were harvested by centrifugation $(4,000 \times g)$ for $45 \mathrm{~min}$ at $4^{\circ} \mathrm{C}$, and stored at $-20^{\circ} \mathrm{C}$.

Purification of ferredoxin and 2-oxoacid:ferredoxin oxidoreductase. All purification steps were performed at $4^{\circ} \mathrm{C}$. A $15 \mathrm{~g}$ batch of frozen cells was thawed and resuspended in $10 \mathrm{mM}$ sodium phosphate buffer ( $\mathrm{pH} 7.0)$ (buffer A) to produce a $20 \%$ (wt/vol) homogeneous solution. Cells were disrupted by passing them three times through a chilled FRENCH Pressure Cell (Thermo Spectronic Inc.) at a pressure of 1,200 psi. Cell debris was precipitated by centrifugation $(150,000 \times g)$ for $90 \mathrm{~min}$ at $4^{\circ} \mathrm{C}$. Precipitates were composed of two layers. The soft upper layer was carefully collected and resuspended in buffer $\mathrm{A}$, and then recentrifuged using the same conditions. Supernatant fractions after centrifugation were combined and dialyzed three times against 5 liters of buffer A for $24 \mathrm{~h}$ to remove salts and to enhance the recovery of iron-sulfur proteins during the DEAE-Sephacel column chromatography purification step. The dialyzed solutions are referred to as cell extracts.

Ferredoxin was purified by comparing the absorbances of collected column chromatography fractions at $283 \mathrm{~nm}$ and $405 \mathrm{~nm}$. Briefly, cell extract was applied to a column of DEAE-Sephacel $(3.0 \times 20 \mathrm{~cm})$ equilibrated with buffer A. The column was then washed with $500 \mathrm{ml}$ of buffer A until absorbance at $280 \mathrm{~nm}$ reached the base line, and was then eluted with $400 \mathrm{ml}$ of a linear gradient of 0 to $0.15 \mathrm{M} \mathrm{NaCl}$ in buffer $\mathrm{A}$. If the brown colored sample remained in the column, further elution was carried out with $300 \mathrm{ml}$ of $0.15 \mathrm{M} \mathrm{NaCl}$ in buffer A. Fractions containing the dark brown ferredoxin were pooled, adjusted to $1 \mathrm{M}$ using powdered ammonium sulfate, and then loaded onto a Phenyl Sepharose column $(1.5 \times 15 \mathrm{~cm})$ equilibrated with buffer A containing $1 \mathrm{M}$ ammonium sulfate. The column was then washed using $200 \mathrm{ml}$ of the same equilibration buffer and eluted with $300 \mathrm{ml}$ of a reverse linear gradient of 1 to $0 \mathrm{M}$ ammonium sulfate in buffer $\mathrm{A}$. The fractions containing ferredoxin were collected and dialyzed three times against 5 liters of buffer $\mathrm{A}$ for $24 \mathrm{~h}$ to remove the ammonium sulfate. The dialyzed protein solution was then applied to a QSepharose column $(1.5 \times 15 \mathrm{~cm})$ equilibrated with buffer A, washed with $200 \mathrm{ml}$ of buffer A, and eluted with a $300 \mathrm{ml}$ linear gradient of 0 to $0.3 \mathrm{M} \mathrm{NaCl}$ in buffer $\mathrm{A}$. The collected protein solution, containing ferredoxin, was concentrated by using an Amicon concentrator (Amicon Centriprep YM-10, Millipore), and the concentrated sample was applied to a Sephadex G-50 column $(1.5 \times 50 \mathrm{~cm})$ equilibrated with buffer A containing $20 \mathrm{mM} \mathrm{NaCl}$ and eluted with the same equilibration buffer. Fractions containing ferredoxin were collected and dialyzed twice against $5 \mathrm{~L}$ of buffer $\mathrm{A}$ to remove salt. Dialyzed protein solutions are referred to as purified ferredoxin solutions, and if required, were concentrated using an Amicon concentrator (Amicon Centriprep YM-10, Millipore).

To purify 2-oxoacid:ferredoxin oxidoreductase, cell extract was applied to a DEAE-Sephacel column $(3.0 \times 20 \mathrm{~cm})$ equilibrated with buffer A. The column was then washed with $300 \mathrm{ml}$ of buffer 
A until the absorbance at $280 \mathrm{~nm}$ reached base line, and then eluted with $400 \mathrm{ml}$ of a linear gradient of 0 to $0.15 \mathrm{M} \mathrm{NaCl}$ in buffer $\mathrm{A}$. Fractions containing 2-oxoacid:ferredoxin oxidoreductase activity were pooled, powdered ammonium sulfate was added to the pooled protein solution to $0.5 \mathrm{M}$, which was then loaded onto a Phenyl Sepharose column $(1.5 \times 15 \mathrm{~cm})$ equilibrated with buffer A containing $0.5 \mathrm{M}$ ammonium sulfate. The column was then washed with $200 \mathrm{ml}$ of the same equilibration buffer and eluted with $300 \mathrm{ml}$ of a reverse linear gradient of 0.5 to $0 \mathrm{M}$ ammonium sulfate in buffer $\mathrm{A}$. Fractions containing 2-oxoacid:ferredoxin oxidoreductase activity were collected, and concentrated using an Amicon concentrator (Amicon Centriprep YM-10, Millipore). The concentrated protein solution was then applied to a Superdex 200 column $(1.5 \times 60 \mathrm{~cm})$ equilibrated with buffer A containing $0.2 \mathrm{M} \mathrm{NaCl}$ and eluted with the same equilibration buffer. Fractions containing 2-oxoacid:ferredoxin oxidoreductase activity were collected and dialyzed three times against 5 liters of buffer A for $24 \mathrm{~h}$ to remove salt. The dialyzed protein solution was then applied to a Q-Sepharose column $(1.5 \times 15 \mathrm{~cm})$ equilibrated with buffer A, washed with $200 \mathrm{ml}$ of buffer $\mathrm{A}$, and then eluted with $300 \mathrm{ml}$ of a linear gradient of 0 to $0.3 \mathrm{M} \mathrm{NaCl}$ in buffer A. Fractions containing 2-oxoacid:ferredoxin oxidoreductase activity were collected and dialyzed as described above. The dialyzed sample was then loaded onto a Blue Sepharose column $(1.0 \times 10 \mathrm{~cm})$ equilibrated with buffer $\mathrm{A}$ and eluted with $300 \mathrm{ml}$ of a linear gradient of 0 to $0.3 \mathrm{M} \mathrm{NaCl}$ in buffer A. Fractions containing 2-oxoacid:ferredoxin oxidoreductase activity were collected and then dialyzed as described above. Dialyzed protein solutions are referred to as purified 2-oxoacid:ferredoxin oxidoreductase solutions.

Measurement of enzymatic activity. Standard 2-oxoacid:ferredoxin oxidoreductase activity assays were carried out at $55^{\circ} \mathrm{C}$ by monitoring changes in absorbance at $550 \mathrm{~nm}$, due to the ferredoxindependent reduction of a horse heart cytochrome $\mathrm{c}$ in the presence of 2-oxoglutarate as a substrate, by using a modified version of the method described by Kerscher et al. (Kerscher et al., 1982; Iwasaki and Oshima, 2001). Briefly, the enzyme reaction was started by adding $0.1 \mathrm{ml}$ of the purified enzyme solution $(50 \mu \mathrm{g} / \mathrm{ml})$ to $0.1 \mathrm{ml}$ of prewarmed purified ferredoxin solution $(260 \mu \mathrm{g} / \mathrm{ml})$ and $0.8 \mathrm{ml}$ of prewarmed $100 \mathrm{mM}$ sodium phosphate buffer $(\mathrm{pH} 7.0)$ containing $20 \mu \mathrm{M}$ horse heart cytochrome c, $20 \mu \mathrm{M}$ coenzyme A, and $4 \mathrm{mM}$ 2-oxoglutarate. For all measurement, the effect of cytochrome c nonenzymatic reduction was taken into consideration and subtracted from values determined in the presence of enzyme. Measurements were carried out at least three times. Standard deviations never exceeded $10 \%$ of the mean values. One enzyme unit was defined as the amount of enzyme required to reduce $1 \mu \mathrm{mol}$ of cytochrome c per min at $55^{\circ} \mathrm{C}$. A reduction of cytochrome c by $1 \mathrm{nmol}$ corresponded to an $A_{550 \mathrm{~nm}}$ increase of 0.021 (Kerscher et al., 1982). To determine substrate specificities and the effects of ferredoxin on the activities of purified enzyme toward other kinds of 2-oxoacids, namely, glyoxylate, pyruvate, and 2-oxobutyrate, in addition to 2-oxoglutarate were also examined in the absence and presence of ferredoxin (Zhang et al., 1996).

Effects of $\mathbf{p H}$ and temperature. The optimum $\mathrm{pH}$ and temperature for purified 2-oxoacid:ferredoxin oxidoreductase activity were determined using a standard 2-oxoacid:ferredoxin oxidoreductase assay using 2-oxoglutarate as a substrate. The effect of $\mathrm{pH}$ on enzyme activity was examined at $55^{\circ} \mathrm{C}$ in the $\mathrm{pH}$ range, 4.0 to 11.0 . The following buffers $(100 \mathrm{mM})$ were used: sodium acetate $(\mathrm{pH} 4.0$ to 6.0 ), sodium phosphate ( $\mathrm{pH} 6.0$ to 8.0 ), Tris/ $\mathrm{HCl}$ ( $\mathrm{pH} 8.0$ to 9.0 ), and sodium bicarbonate ( $\mathrm{pH} 9.0$ to 11.0). The effect of temperature on enzyme activity was investigated at the optimum $\mathrm{pH}(\mathrm{pH}$ 7.0) at temperatures ranging from $10^{\circ} \mathrm{C}$ to $70^{\circ} \mathrm{C}$.

Analytical methods. Absorption spectra were recorded using a Shimadzu UV-2401PC spectrophotometer equipped with a cell holder having a circulating water jacket system. EPR measurements were carried out using a JEOL JES-TE200 spectrometer equipped with a Heli-Tran cryostat system (Model LT-30M, Air Products), in which temperature was monitored using a Scientific Instruments series 5500 temperature indicator/controller (Aasa and Vänngärd, 1975).

Native PAGE (Laemmli, 1970) was performed using a $10 \%$ or $12.5 \%(\mathrm{w} / \mathrm{v})$ gels to assess enzyme purity. Molecular weights of the purified ferredoxin and 2-oxoacid:ferredoxin oxidoreductase from S. solfataricus $\mathrm{P} 1$ were determined using a low molecular weight standard (Amersham Bioscience) on 12.5\% and 10\% SDS-PAGE, respectively (Laemmli, 1970). Gels were stained with silver (Ohsawa and Ebata, 1983). The molecular weights of the native proteins were also determined by HPLC [GPC column (Protein$\mathrm{Pak}^{\mathrm{TM}} 300 \mathrm{SW}, 7.8 \times 300 \mathrm{~mm}$, Waters)] using molecular weight standards (Sigma MW-GF-200 kit) and by matrix-assisted laserdesorption ionization time-of-flight mass spectrometry (MALDITOF-MS, Voyager DE STR, Applied Biosystems) using cytochrome $\mathrm{C}(12 \mathrm{kDa})$ and BSA (66 kDa) for calibration.

$\mathrm{N}$-terminal amino acid sequences were analyzed using an automatic amino acid sequencer (Hewlett Packard protein sequencer HP241) according to the manufacturer's instructions.

Protein concentrations were determined using the Bradford method (Bradford, 1976) using bovine serum albumin as a standard.

\section{Results and Discussion}

Purification and physical properties of ferredoxin from $S$. solfataricus P1. To establish an assay for 2-oxoacid:ferredoxin oxidoreductase from $S$. solfataricus $\mathrm{P} 1$, we purified thermostable ferredoxin from the same strain. The purification procedure described here yielded electrophoretically homogeneous ferredoxin in four steps starting from cell extracts of $S$. solfataricus P1 (DSM1616), as described in "Materials and Methods". Approximately $2.5-4 \mathrm{mg}$ of the purified ferredoxin was routinely obtained from $15 \mathrm{~g}$ batch of frozen cells. The homogeneity of the purified ferredoxin was confirmed on SDS-PAGE gel as a single band of approximately $11 \mathrm{kDa}$ (Fig. 1A). A purity index $\left(A_{408} / A_{280}\right)$ of the purified ferredoxin was 0.71 (Fujii et al., 1991), and its molecular weight was determined using a sizing HPLC column to be approximately $11 \mathrm{kDa}$, indicating that the enzyme was monomeric and showing good agreement with the MALDI-TOF-MS determined molecular mass (Fig. 1B).

Fig. 2 shows optical absorption spectra of the purified ferredoxin $(0.17 \mathrm{mg} / \mathrm{ml}$ in $100 \mathrm{mM}$ sodium phosphate buffer, 


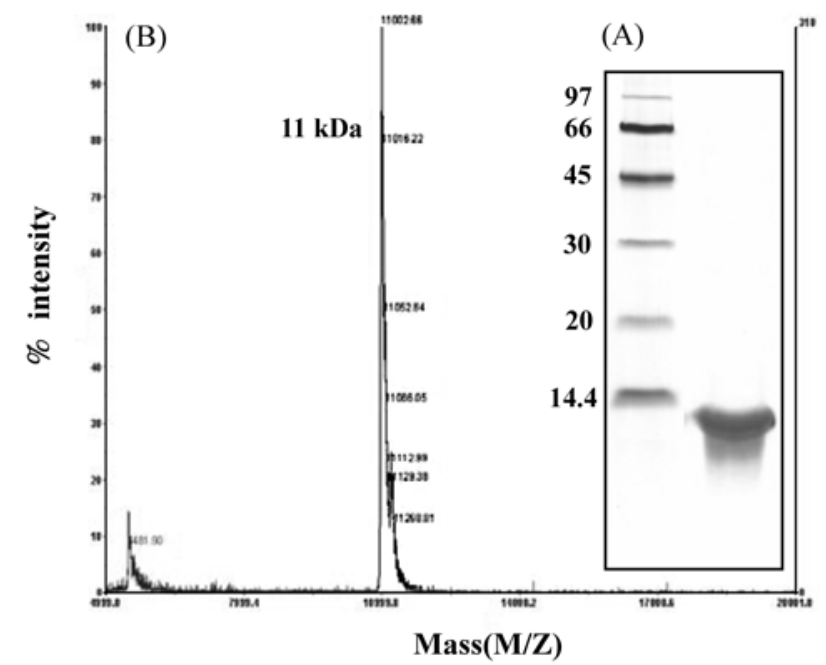

Fig. 1. Electrophoretic and mass spectrometric analyses of the purified ferredoxin from $S$. solfataricus P1. (A) A purified ferredoxin with molecular size markers on a $12.5 \%$ SDS-PAGE gel. (B) MALDI-TOF-MS spectrum of the ferredoxin.

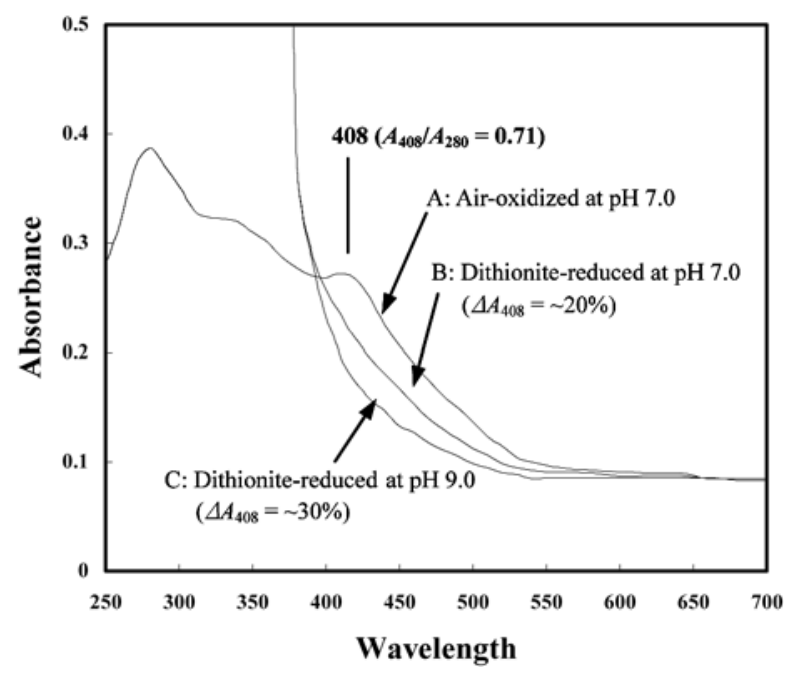

Fig. 2. Ultraviolet-visible absorption spectra of the purified ferredoxin from $S$. solfataricus $\mathrm{P} 1$. Ferredoxin $(0.17 \mathrm{mg} / \mathrm{ml})$ was dissolved in $100 \mathrm{mM}$ sodium phosphate buffer, $\mathrm{pH} 7.0$ (A and B), or in $100 \mathrm{mM}$ Tris-Cl buffer, pH 9.0 (C): A, air-oxidized form as prepared; $\mathrm{B}$ and $\mathrm{C}$, reduced form prepared by adding excess sodium dithionite.

$\mathrm{pH}$ 7.0), which are typical for iron-sulfur containing proteins. Purified ferredoxin displayed an absorption peak at $280 \mathrm{~nm}$ and a broad peak at around $408 \mathrm{~nm}$ in the air-oxidized state (Fig. 2A). The spectrum of dithionite-reduced ferredoxin was obtained by re-scanning after adding an excess of solid sodium dithionite to an air-oxidized sample solution. As shown in Fig. 2B, a ca. $20 \%$ decrease in absorbance was observed at $408 \mathrm{~nm}$. The addition of more sodium dithionite did not cause a further reduction in absorbance at $408 \mathrm{~nm}$. However, the addition of sodium dithionite to an air oxidized

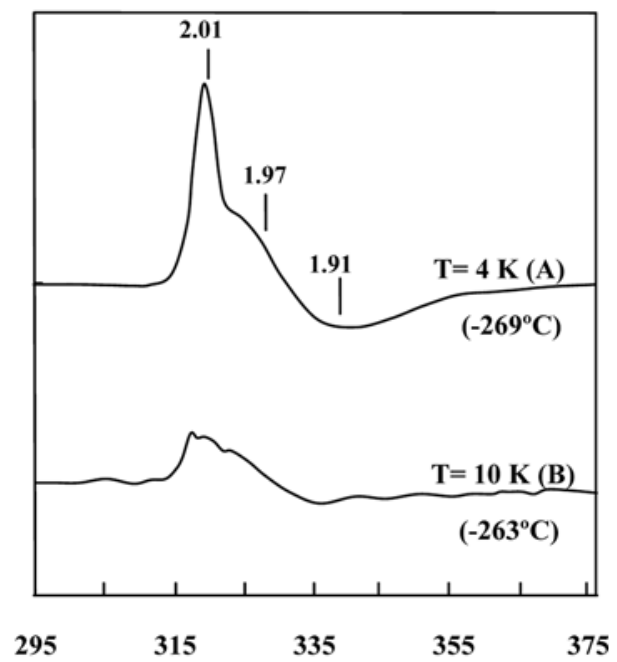

Magnetic Field (mT)

Fig. 3. Temperature dependence by EPR of the air-oxidized form of the purified ferredoxin from $S$. solfataricus P1. The sample was dissolved in $10 \mathrm{mM}$ sodium phosphate buffer ( $\mathrm{pH}$ 7.0). The instrument settings for EPR spectroscopy were; temperature, $4 \mathrm{~K}$ (A) or $10 \mathrm{~K}(\mathrm{~B})$; microwave power $1.02 \mathrm{~mW}$; and modulation amplitude $0.79 \mathrm{mT} ; g$ values are indicated in the figure.

ferredoxin in $100 \mathrm{mM}$ Tris-Cl buffer, $\mathrm{pH}$ 9.0, induced a further reduction at $408 \mathrm{~nm}$ band of approximately $30 \%$ of its original absorbance (Fig. 2C). These observations indicate that the purified ferredoxin was in a partially reduced state under experimental conditions, since most bacterial-type ferredoxins show a reduction of $40 \%$ or more at $400 \mathrm{~nm}$ in the fully reduced state (Blamey et al., 2000; Green et al., 2003). In addition, it appears likely that $S$. solfataricus ferredoxin has an iron-sulfur cluster of unusually low redox potential with $\mathrm{pH}$-dependence. From these results it seems that ferredoxin from $S$. solfataricus $\mathrm{P} 1$ is similar to dicluster-type ferredoxins that possess one low potential iron-sulfur cluster, such as, those from archaea, Sulfolobus sp. strain 7 (Iwasaki et al., 1994), Pyrobaculum islandicum (Nakajima et al., 1998), D. ambivalens (Teixeira et al., 1995), and $S$. acidocaldarius (Breton et al., 1995). The purified ferredoxin showed extreme thermostability, as judged by an unchanged optical absorption spectra after incubation for $2 \mathrm{~h}$ at $75^{\circ} \mathrm{C}$ or for over $48 \mathrm{~h}$ at 55 ${ }^{\circ} \mathrm{C}$ at $\mathrm{pH}$ 7.0. In addition, it is stable at $55^{\circ} \mathrm{C}$ across a broad $\mathrm{pH}$ range, i.e., from 5 to 11 .

Fig. 3 displays the effect of temperature on the X-band EPR spectra of the air-oxidized form of the purified ferredoxin. The EPR spectrum at $4 \mathrm{~K}$ exhibited a sharp peak at $g=2.01$, a broad trough around $g=1.91$ with an extended tail toward higher magnetic field, and a distinct shoulder at $g=1.97$. This single sharp peak, with a $g_{\max }$ of 2.01 , corresponds to a center with an extremely rapid spin relaxation rate. However, this spectrum could not be detected clearly at $10 \mathrm{~K}$. Moreover, the spectral shape was strongly temperature dependent, with noticeable line broadening even at temperatures only slightly 


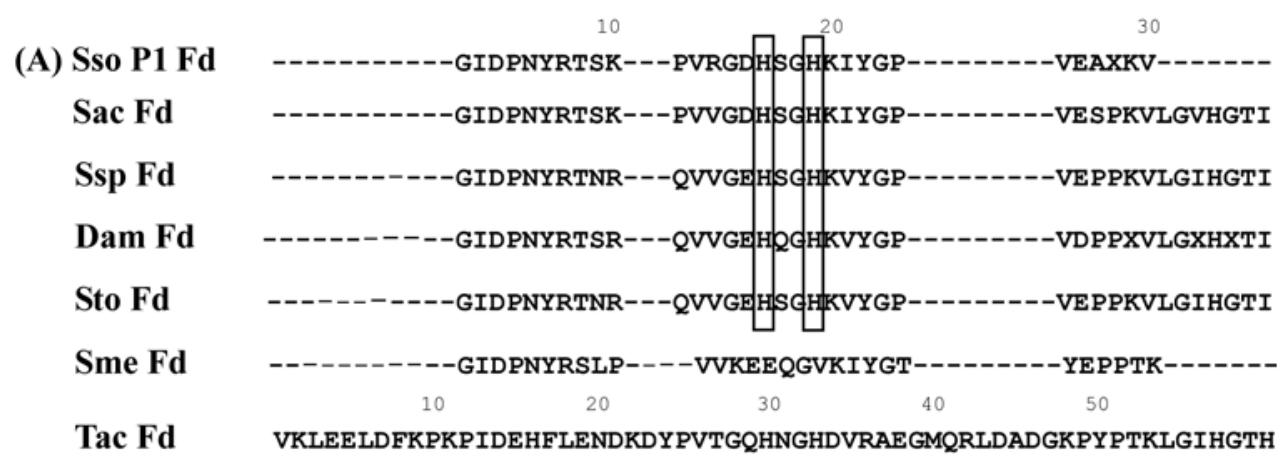

(B) Sso P2 Fd

Fig. 4. Multiple alignments of the N-terminal amino acid sequence of the purified ferredoxin from S. solfataricus P1 (DSM 1616) (Sso P1 Fd) with those of ferredoxins from various other archaea such as Sac (S. acidocaldarius), Ssp (Sulfolobus sp. strain 7), Dam (D. ambivalence), Sto (S. Tokodaii), Sme (S. metallicus), and Tac (T. acidophilum DSM1738) (A), and with that of a putative ferredoxin deduced from genes found in the complete genome of S. solfataricus P2 (Sso P2) (B). The boxes indicated possible ligand residues related to the zinc binding motif.

above $4 \mathrm{~K}$. These results strongly suggest that at least one $[3 \mathrm{Fe}-4 \mathrm{~S}]$ cluster is present in the purified ferredoxin. Similar signals were observed for the [3Fe-4S] cluster of dicluster $(7 \mathrm{Fe})$ ferredoxins from Sulfolobus sp. strain 7 (Iwasaki et al., 1994), S. acidocaldarius (Breton et al., 1995), Thermus thermophilus (Hagen et al., 1985), and D. ambivalens (Teixeira et al., 1995).

The sequence of 30 amino acid residues at the N-terminus of the purified ferredoxin from S. solfataricus P1 was determined and compared with the sequence data retrieved from the Entrez server at NCBI (http//www.ncbi.nlm.nih.gov/ Entrez/) and from papers on ferredoxins from other archaea (Fig. 4). The N-terminal amino acid sequence of ferredoxin from S. solfataricus P1 (DSM 1616) showed highest similarity (93\% identity) with that of $S$. acidocaldarius ferredoxin containing diclusters, [3Fe-4S] [4Fe-4S] (Minami et al., 1985; Breton et al., 1995). In addition, it displayed more than 50\% similarity with ferredoxins containing dicluster, [3Fe-4S] [4Fe-4S], from Sulfolobus sp. strain 7, D. ambivalens, Sulfolobus Tokodaii, and Sulfolobus metallicus belonging to the Sulfolobaceae family (Wakagi et al., 1996; Iwasaki et al., 1997; Gomes et al., 1998; Iwasaki and Oshima, 2001). However, it showed little similarity with dicluster containing ferredoxins, [3Fe-4S] [4Fe-4S], from T. acidophilum or single cluster containing ferredoxins, $[4 \mathrm{Fe}-4 \mathrm{~S}]$, from $P$. furiosus, which belong to other thermophilic archaea (Wakagi et al., 1996; Gomes et al., 1998). The N-terminal sequence of ferredoxin from S. solfataricus $\mathrm{P} 1$ was not identical to that of a putative ferredoxin composed of 103 amino acids derived from the genomic sequence of $S$. solfataricus $\mathrm{P} 2$ (She et al., 2001 ); these shared a similarity of $69 \%$. In addition, the purified ferredoxin appears to be a zinc-containing protein, because it contains signature histidine residues in its $\mathrm{N}$ terminal sequence, like the amino acid sequences of other zinc-containing ferredoxins (Iwasaki et al., 1997).

Purification and physical properties of 2-oxoacid:ferredoxin oxidoreductase from $\boldsymbol{S}$. solfataricus $\mathbf{P 1}$. As shown in Table 1, 2-oxoacid:ferredoxin oxidoreductase was purified to electrophoretic homogeneity using five steps starting from $S$. solfataricus $\mathrm{P} 1$ (DSM1616) cell extract, as described in "Materials and Methods". The 2-oxoacid:ferredoxin oxidoreductase was purified 44-fold with a yield of $6 \%$, and showed a specific activity of $52.6 \mathrm{units} / \mathrm{mg}$ for the reduction of cytochrome $\mathrm{c}$ at $55^{\circ} \mathrm{C}(\mathrm{pH} 7.0)$ by standard enzyme assay using 2-oxoglutarate

Table 1. Summary of the purity of the 2-oxoacid:ferredoxin oxidoreductase from $S$. solfataricus P1

\begin{tabular}{lccccc}
\hline \multicolumn{1}{c}{ Step } & $\begin{array}{c}\text { Protein } \\
(\mathrm{mg})\end{array}$ & $\begin{array}{c}\text { Activity } \\
(\mathrm{U})^{*}\end{array}$ & $\begin{array}{c}\text { Specific activity } \\
(\mathrm{U} / \mathrm{mg})\end{array}$ & $\begin{array}{c}\text { Yield } \\
(\%)\end{array}$ & $\begin{array}{c}\text { Purification } \\
\text { (fold) }\end{array}$ \\
\hline Crude & 137.7 & 167.1 & 1.2 & 100 & 1 \\
DEAE-Sepharose & 27 & 149.9 & 5.5 & 89 & 5 \\
Phenyl- Sepharose & 4 & 110.5 & 30.2 & 66 & 25 \\
Superdex-200 & 2 & 63.1 & 35.7 & 38 & 29 \\
Q- Sepharose & 1 & 37.9 & 37.9 & 23 & 31 \\
Blue- Sepharose & 0.2 & 10.5 & 52.6 & 6 & 44 \\
\hline
\end{tabular}

*One unit of the enzyme is defined as the amount of enzyme required to reduce $1 \mu \mathrm{mol}$ of cytochrome c per min at $55^{\circ} \mathrm{C}$. A cytochrome c reduction of $1 \mathrm{nmol}$ corresponded to an $A_{550 \mathrm{~nm}}$ increase of 0.021 . 


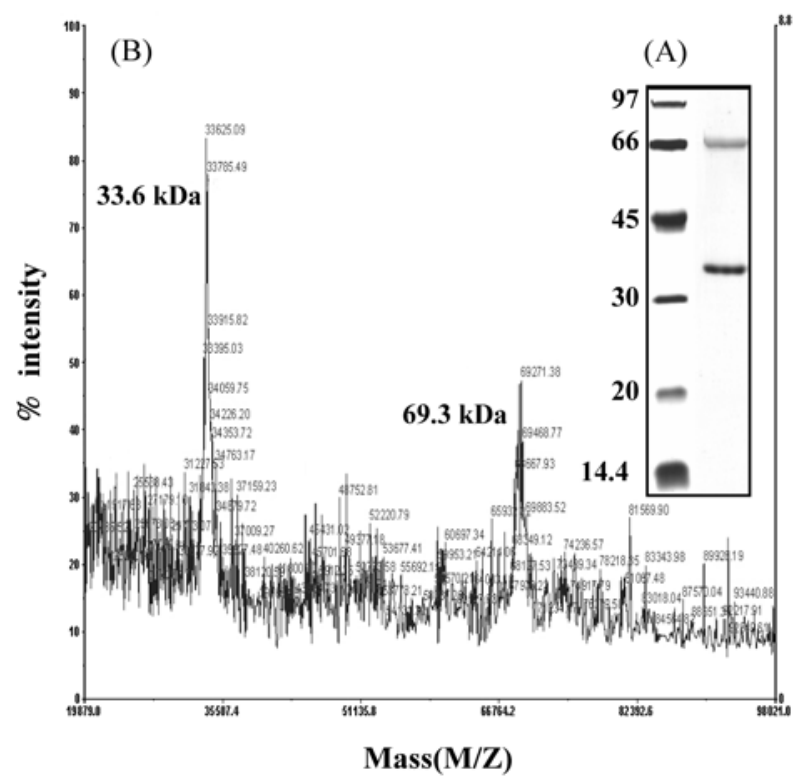

Fig. 5. Electrophoretic and mass spectrometric analyses of the purified 2-oxoacid:ferredoxin oxidoreductase from $S$. solfataricus P1. (A) A purified 2-oxoacid:ferredoxin oxidoreductase with molecular size markers on a $10 \%$ SDS-PAGE gel. (B) MALDITOF-MS spectrum of 2-oxoacid:ferredoxin oxidoreductase.

and the purified ferredoxin from $S$. solfataricus P1 (DSM1616) as substrates. The homogeneity of the purified enzyme was confirmed on native PAGE gel as a single band and by the presence of a single peak at approximately $103 \mathrm{kDa}$ by HPLC using a sizing column (data not shown). The molecular size of the enzyme is similar to those of other 2-oxoacid:ferredoxin oxidoreductases from hyperthermophilic archaea such as Sulfolobus sp. strain 7 (103 kDa), P. furiosus $(115 \mathrm{kDa})$, and Archaeoglobus fulgidus $(120 \mathrm{kDa})$ and corresponds roughly to half the size of that of Halobacterium halobium enzyme (Kerscher and Oesterhelt, 1981; Zhang et al., 1996). The purified enzyme was analyzed by SDS-PAGE gel as a heterodimeric enzyme, with apparent molecular masses of $34 \mathrm{kDa}$ and $69 \mathrm{kDa}$, respectively (Fig. 5A). Moreover, the molecular masses of these two subunits are in good agreement with those obtained by MALDI-TOF-MS analysis (Fig. 5B). The 2-oxoacid:ferredoxin oxidoreductase from Sulfolobus sp. strain $7(103 \mathrm{kDa})$ was previously reported to be a heterodimeric enzyme composed of 37 and $70 \mathrm{kDa}$ (Zhang et al., 1996). In contrast, 2-oxoacid:ferredoxin oxidoreductases from other hyperthermophilic archaea such as $P$. furiosus (Zhang et al., 1996; Kletzin and Adams, 1996), A. fulgidus (Kunow et al., 1995), and H. halobium (Kerscher and Oesterhelt, 1981; Zhang et al., 1996) have been reported as heterotetrameric enzymes.

The effect of $\mathrm{pH}$ and temperature on 2-oxoacid:ferredoxin oxidoreductase activity was determined using cytochrome $\mathrm{c}$ at several different $\mathrm{pHs}$ and temperatures. The purified enzyme displayed maximum activity at $\mathrm{pH}$ 7.0-8.0 (Fig. 6A) and at

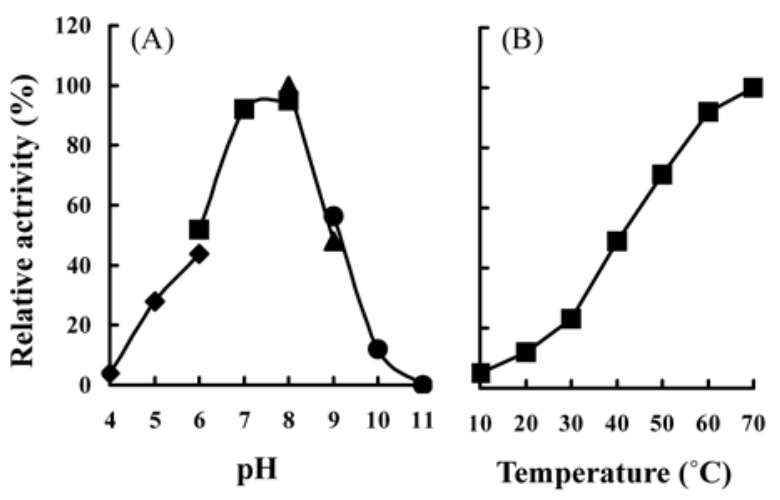

Fig. 6. Effect of $\mathrm{pH}$ and temperature on the activity of the purified 2-oxoacid:ferredoxin oxidoreductase. The effects of $\mathrm{pH}$ and temperature on the activity of the purified 2-oxoacid:ferredoxin oxidoreductase were determined spectrophotometrically using a standard 2-oxoacid:ferredoxin oxidoreductase assay using 2-oxoglutarate as a substrate, as described in "Materials and Methods". The enzyme activity was examined at $55^{\circ} \mathrm{C}$ and different pH's (A) or at $\mathrm{pH} 8.0$ and different temperatures (B). Required $\mathrm{pH}$ 's were obtained using $100 \mathrm{mM}$ sodium citrate buffer $(\boldsymbol{)})$ in the $\mathrm{pH}$ range 5.0 to $6.0 ; 100 \mathrm{mM}$ sodium phosphate buffer $(\boldsymbol{\square})$ in the $\mathrm{pH}$ range 6.0 to $8.0 ; 100 \mathrm{mM}$ Tris-Cl buffer $(\boldsymbol{\Delta})$ in the $\mathrm{pH}$ range 8.0 to 9.0 , and $100 \mathrm{mM}$ sodium bicarbonate buffer $(\boldsymbol{O})$ in the $\mathrm{pH}$ range 9.0 to 11.0 .

$70^{\circ} \mathrm{C}$ (Fig. 6B). Activity assays were routinely performed for convenience using phosphate buffer, $\mathrm{pH} 7.0$, and at $55^{\circ} \mathrm{C}$, to characterize the purified enzyme, since horse heart cytochrome $\mathrm{c}$ is stable to $55^{\circ} \mathrm{C}$.

The dependence of the purified enzyme on ferredoxin obtained from $S$. solfataricus $\mathrm{P} 1$ and its substrate specificity toward various 2-oxoacids, such as pyruvate, 2-oxobutyrate, and 2-oxoglutarate were investigated; results are summarized in Tables 2 and 3. As shown in Table 2, the cytochrome c reducing activity of the enzyme was negligible toward any of the 2-oxoacids in the absence of ferredoxin. Cytochrome $\mathrm{c}$ reducing activities toward these 2-oxoacids in the presence of cognate ferredoxin followed the order of 2-oxoglutarate $>$ pyruvate $>2$-oxobutyrate. Moreover, its enzymatic activity for 2-oxoglutarate was about three times higher than that for pyruvate, and about five times higher than that for 2oxobutyrate. In contrast, no activity was detected toward glyoxylate either in the presence or absence of ferredoxin. These results indicate that the purified enzyme depends on ferredoxin for 2-oxoacid-dependent cytochrome c reduction and that it shows broad substrate specificity toward various 2oxoacids. Our results are in agreement with a report that the homologous enzyme from Sulfolobus sp. strain 7 also depends on ferredoxin for cytochrome c reduction, but contrast with a report that the specific activities of the enzyme in the presence of cognate ferredoxin follow the order; 2-oxoglutarate $>2$ oxobutyrate $>$ pyruvate (Zhang et al., 1996). In addition, pyruvate:ferredoxin oxidoreductase from $H$. halobium accepts 2-oxobutyrate, pyruvate, and 2-oxoglutarate; in decreasing 
Table 2. Effect of ferredoxin on 2-oxoacid:ferredoxin oxidoreductase activity

\begin{tabular}{|c|c|c|c|}
\hline \multirow{2}{*}{$\begin{array}{c}\mathrm{O} \\
\| \\
\text { R-C-COO- } \\
\text { Alkyl group }\end{array}$} & \multirow[t]{2}{*}{ Substrate $(4 \mathrm{mM})$} & \multicolumn{2}{|c|}{ Relative activity of cytochrome c reduction (\%) } \\
\hline & & - ferredoxin & $+25 \mu \mathrm{M}$ ferredoxin \\
\hline$-\mathrm{H}$ & Glyoxylate & $\sim 1$ & $\sim 1$ \\
\hline$-\mathrm{CH}_{3}$ & Pyruvate & 4.3 & 31.2 \\
\hline$-\mathrm{CH}_{2}-\mathrm{CH}_{2}-\mathrm{CH}_{3}$ & 2-Oxobutyrate & 4.1 & 20.4 \\
\hline$-\mathrm{CH}_{2}-\mathrm{CH}_{2}-\mathrm{COO}^{-}$ & 2-Oxoglutarate & 4.5 & 100.0 \\
\hline
\end{tabular}

The ferredoxin dependence of the 2-oxoacid:ferredoxin oxidoreductase reaction was examined using a standard assay in either the absence or presence $(25 \mu \mathrm{M})$ of ferredoxin as described in "Materials and Methods".

Table 3. Kinetic parameters of 2-oxoacid:ferredoxin oxidoreductase for various 2-oxoacids

\begin{tabular}{lccc}
\hline & $K_{m}(\mathrm{M})$ & $k_{\text {cat }}\left(\mathrm{min}^{-1}\right)$ & $k_{\text {cat }} / K_{m}\left(\mathrm{M}^{-1} \cdot \mathrm{min}^{-1}\right)$ \\
\hline 2-Oxoglutarate & $1.63 \times 10^{-4}$ & $4.52 \times 10^{2}$ & $2.77 \times 10^{6}$ \\
Pyruvate & $2.75 \times 10^{-4}$ & $1.44 \times 10^{2}$ & $5.24 \times 10^{5}$ \\
2-Oxobutyrate & $5.16 \times 10^{-4}$ & $0.93 \times 10^{2}$ & $1.80 \times 10^{5}$ \\
\hline
\end{tabular}

order of specific activity (Kerscher and Oesterhelt, 1981). The substrate specificities of the purified enzyme toward various 2-oxoacids are summarized in Table 3 . The highest $K_{m}$ value of $516 \mu \mathrm{M}$ was observed for 2-oxobutyrate, whereas the lowest $K_{m}$ value of $163 \mu \mathrm{M}$ was observed for 2-oxoglutarate. However, $k_{\text {cat }}$ was highest for 2-oxoglutarate and lowest for 2oxobutyrate. The specificity constant $\left(k_{\text {cat }} / K_{m}\right)$ values for 2 oxoglutarate, 2-oxobutyrate, and pyruvate were $2.77 \times 10^{6}$, $5.24 \times 10^{5}$, and $1.80 \times 10^{5} \mathrm{M}^{-1} \cdot \mathrm{min}^{-1}$, respectively. Moreover, the turnover of 2-oxoglutarate was 5 times that of pyruvate and more than 10 times that of 2-oxobutyrate. Our data indicate that the best 2-oxoacid substrate (from among the three tested) for 2-oxoacid:ferredoxin oxidoreductase from $S$. solfataricus $\mathrm{P} 1$ is 2-oxoglutarate.

In conclusion, the present paper reports for the first time on the purification and characterization of thermostable ferredoxin and 2-oxoacid:ferredoxin oxidoreductase from $S$. solfataricus P1 strain. $S$. solfataricus ferredoxin was found to contain a dicluster, i.e., a [3Fe-4S] cluster and a [4Fe-4S] cluster, by spectroscopic analyses, and to possibly contain one zinc center by comparing its N-terminal amino acid sequence with those of well known zinc-containing ferredoxins. In addition, it is suggested that this $S$. solfataricus 2-oxoacid:ferredoxin oxidoreductase is $\alpha \beta$-type heterodimeric enzyme and shows broad substrate specificity toward 2-oxoacids, but a fastest turnover of 2-oxoglutarate. This new information should be useful for those performing structural studies on these two proteins and the study on the mechanism of electron transfer between ferredoxin and 2-oxoacid:ferredoxin oxidoreductase.

Acknowledgments We thank the Central Laboratory at Kangwon National University for performing the Erdman degradation required to elucidate the amino acid sequence analysis and for performing the MALDI-TOF-MS.

\section{References}

Aasa, R. and Vänngärd, T. (1975) EPR signal intensity and powder shapes: a re-examination. J. Magn. Reson. 19, 308-315.

Aono, S., Bryant, F. O. and Adams, M. W. W. (1989) A novel and remarkably thermostable ferredoxin from the hyperthermophilic archaebacterium Pyrococcus furiosus. J. Bacteriol. 171, 34333439.

Beinert, H. (1990) Recent developments in the field of iron-sulfur proteins. FASEB J. 4, 2483-2491.

Beinert, H., Kennedy, M. C. and Stout, C. D. (1996) Aconitase as iron-sulfur protein, enzyme, and iron-regulatory protein. Chem. Rev. 96, 2335-2374.

Blamey, J. M. and Adams, M. W. W. (1993) Purification and characterization of pyruvate ferredoxin oxidoreductase from the hyperthermophilic archeon Pyrococcus furiosus. Biochim. Biophys. Acta. 1161, 19-27.

Blamey, J. M., Chiong, M., López, C. and Smith, E. T. (2000) Purification and characterization of ferredoxin from hyperthermophilic Pyrococcus woesei. Anaerobe 6, 285-290.

Bradford, M. M. (1976) A rapid and sensitive method for the quantitation of microgram quantities of protein utilizing the principle of protein-dye binding. Anal. Biochem. 72, 248-254.

Breton, J. L., Duff, J. L. C., Butt, J. N., Armstrong, F. A., George, S. J., Petillot, Y., Forest, E., Schafer, G. and Thompson, A. J. (1995) Identification of the iron-sulfur clusters in a ferredoxin from the archaeon Sulfolobus acidocaldarius. Evidence for a reduced $[3 \mathrm{Fe}-4 \mathrm{~S}]$ cluster with $\mathrm{pH}$-dependent electronic properties. Eur. J. Biochem. 233, 937-946.

Brock, T. D., Brock, K. M., Belly, R. T. and Weiss, R. L. (1972) Sulfolobus: a new genus of sulfur oxidizing bacteria living at low $\mathrm{pH}$ and high temperature. Arch. Microbiol. 84, 54-68.

Broderick, J. B., Duderstadt, R. E., Fernandez, D. C., Wojtuszewski, K., Henshaw, T. F. and Johnson, M. K. (1997) Pyruvate formate-lyase activating enzyme is an iron-sulfur protein. J. Am. Chem. Soc. 119, 7396-7397.

Brostedt, E. and Nordlund, S. (1991) Purification and partial 
characterization of a pyruvate oxidoreductase from the photosynthetic bacterium Rhodospirillum rubrum grown under nitrogen fixing conditions. Biochem. J. 279, 155-158.

Daas, P. J., Hagen, W. R., Keltjens, J. T. and Vogels, G. D. (1994) Characterization and determination of the redox properties of the $2[4 \mathrm{Fe}-4 \mathrm{~S}]$ ferredoxin from Methanosarcina barkeri strain MS. FEBS Lett. 356, 342-344.

Fujii, T., Moriyama, H., Takenaka, A., Tanaka, N., Wakagi, T. and Oshima, T. (1991) Crystallization and preliminary X-ray studies on Sulfolobus acidocaldarius ferredoxin. J. Biochem. (Tokyo) 110, 472-473.

Gomes, C. M., Faria, A., Carita, J. C., Mendes, J., Regalla, M., Chicau, P., Huber, H., Stetter, K. O. and Teixeira, M. (1998) Di-cluster, seven-iron ferredoxins from hyperthermophilic Sulfolobales. JBIC 3, 499-507.

Grandoni, J. A., Switzer, R. L., Makaroff, C. A. and Zalkin, H. (1989) Evidence that the iron-sulfur cluster of Bacillus subtilis glutamine phosphoribosylpyrophosphate amidotransferase determines stability of the enzyme to degradation in vivo. $J$. Biol. Chem. 264, 6058-6064.

Green, A. J., Munro, A. W., Cheesman, M. R., Reid, G. A., Wachenfeldt, C. and Chapman, S. K. (2003) Expression, purification and characterization of a Bacillus subtilis ferredoxin: a potential electron transfer donor to cytochrome P450 Biol. J. Inorg. Biochem. 93, 92-99.

Grinberg, A. V., Hannemann, F., Schiffler, B., Muller, J., Heinemann, U. and Bernhardt, R. (2000) Adrenodoxin: Structure, stability, and electron transfer properties. Proteins $\mathbf{4 0}$, 590-612.

Hagen, W. R., Dunham, W. R., Johnson, M. K. and Fee, J. A. (1985) Quarter field resonance and integer-spin/half-spin interaction in the EPR of Thermus thermophilus ferredoxin. Possible new fingerprints for three iron clusters. Biochim. Biophys. Acta 828, 369-374

Hentze, W. and Kunh, L. C. (1996) Molecular control of vertebrate iron metabolism: mRNA-based regulatory circuits operated by iron, nitric oxide, and oxidative stress. Proc. Natl. Acad. Sci. USA 93, 8175-8182.

Howard, J. B. and Rees, D. C. (1996) Structural basis of biological nitrogen fixation. Chem. Rev. 96, 2965-2982.

Iwasaki, T. and Oshima, T. (2001) Ferredoxin and related enzymes from Sulfolobus; in Methods in Enzymology 334, Adams, M. W. W. (ed.), pp. 3-22, Academic Press, New York, USA.

Iwasaki, T., Suzuki, T., Kon, T., Imai, T., Urushiyama, A., Ohmori, D. and Oshima, T. (1997) Novel zinc-containing ferredoxin family in thermoacidophilic archaea. J. Biol. Chem. 272, 3453-3458.

Iwasaki, T., Wakagi, T., Isogani, Y., Tanaka, K., Lizuka, T. and Oshima, T. (1994) Functional and evolutionary implications of a $[3 \mathrm{Fe}-4 \mathrm{~S}]$ cluster of the dicluster-type ferredoxin from the thermoacidophilic archaeon, Sulfolobus sp. strain 7. J. Biol. Chem. 269, 29444-29450.

Johnson, M. K. (1994) Iron-Sulfur Proteins; in Encyclopedia of Inorganic Chemisty, R. B. King (Ed.), pp. 1896-1915, WileyInterscience, New York, USA.

Kerscher, L., Nowitzki, S. and Oesterhelt, D. (1982) Thermophilic archaebacteria contain bacterial-type ferredoxins acting as electron acceptors of 2-oxoacid:ferredoxin oxidoreductases. Eur. J. Biochem. 128, 223-230.

Kerscher, L. and Oesterhelt, D. (1981) Purification and properties of two 2-oxoacid:ferredoxin oxidoreductases from Halobacterium halobium. Eur. J. Biochem. 116, 587-594.

Kletzin, A. and Adams, M. W. W. (1996) Molecular and phylogenetic characterization of pyruvate and 2-ketoisovalerate ferredoxin oxidoreductases from Pyrococcus furiosus and pyruvate ferredoxin oxidoreductase from Thermotoga martima. J. Bacteriol. 178, 248-257.

Koike, M. and Koike, K. (1976) Structure, assembly and function of mammalian $\alpha$-keto acid dehydrogenase complexes. $A d v$. Biophys. 9, 187-227.

Kounosu, A., Li, Z., Cosper, N. J., Shokes, J. E., Scott, R. A., Imai, T., Urushiyama, A. and Iwasaki, T. (2004) Engineering a three-cystein, one-histidine ligand environment into a new hyperthermophilic archaeal Rieske-type [2Fe-2S] ferredoxin from Sulfolobus solfataricus. J. Biol. Chem., 279, 12519-12528.

Kunow, J., Linder, D. and Thauer, R. K. (1995) Pyruvate:ferredoxin oxidoreductase from the sulfate-reducing Achaeoglobus fulgidus: molecular composition, catalytic properties, and sequence alignments. Arch. Microbiol. 163, 2128.

Laemmli, U. K. (1970) Cleavage of structural proteins during the assembly of the head of bacteriophage T4. Nature 227, 680685.

Matsubara, H. and Saecki, K. (1992) Structural and functional diversity of ferredoxins and related proteins. Adv. Inorg. Chem. 38, 223-280.

Meinecke, B., Bertram, J. and Gottachalk, G. (1989) Purification and characterization of the puruvate-ferredoxin oxidoreductase from Clostridium acetobutylicum. Arch. Microbiol. 152, 244250.

Michaels, M. L., Pham, L., Nghiem, Y., Cruz, C. and Miller, J. H. (1990) MutY, an adenine glycosylase active on G-A mispairs, has homology to endonuclease III. Nucleic Acids Res. 18, 3841-3845.

Minami, Y., Wakabayashi, S., Wada, K., Matsubara, H., Kerscher, L. and Oesterhelt, D. (1985) J. Biochem. (Tokyo) 97, 745-751.

Mortenson, L. E., Valentine, R. C. and Carnahan, J. E. (1962) An electron transport factor from Clostridium pasteurianum. Biochem. Biophys. Res. Commun. 7, 448-452.

Nakajima, Y., Fujiwara, T. and Fukumori, Y. (1998) Purification and characterization of a $[3 \mathrm{Fe}-4 \mathrm{~S}][4 \mathrm{Fe}-4 \mathrm{~S}]$ type ferredoxin from hyperthermophilic archeon, Pyrobaculum islandicum. $J$. Biochem. 23, 521-527.

Ohsawa, K. and Ebata, N. (1983) Silver stain for detecting 10femtogram quantities of protein after polyacrylamide gel electrophoresis. Anal. Biochem. 135, 409-415.

Rosa, M. D., Gambacorta, A. and Bu'lock, J. D. (1975) Extremely thermophilic acidophilic bacteria convergent with Sulfolobus acidocaldarius. J. Gen. Microbiol. 86, 156-164.

She, Q., Singh, R. K., Confalonieri, F., Zivanovic, Y., Allard, G., Awayez, M. J., Chan Weiher, C. C. Y., Clausen, I. G., Curtis, B. A., Moors, A. D., Erauso, G., Fletcher, C., Gordon, P. M. K., Heikamp, D. J. I., Jeffries, A. C., Kozera, C. J., Medina, N., Peng, X., Thi-Ngoc, H. P., Redder, P., Schenk, M. E., Theriault, C., Tolstrup, N., Charlebois, R. L., Doolittle, W. F., Duguet, M., Gaasterland, T., Garrett, R. A., Ragan, M. A., Sensen, C. W. and Oost, V. D. J. (2001) The complete genome of the crenarchaeon Sulfolobus solfataricus P2. Proc. Natl. Acad. Sci. USA 98, 7835-7840.

Teixeira, M., Batista, R., Campos, A. P., Gomes, C., Mendes, J., 
Pacheco, I., Anemuller, S. and Hagen, W. R. (1995) A seveniron ferredoxin from the thermoacidophilic archaeon Desulfurolobus ambivalens. Eur. J. Biochem. 227, 322-327.

Thauer, R. K. and Schonheit, P. (1982) Iron-sulfur complexs of ferredoxin as a storage form of iron in Clostridium pasteurianum; in Iron-Sulfur Proteins. T. G. Spiro (Ed.), pp. 329-341, Wiley-Interscience, New York, USA.

Wakagi, T., Fujii, T. and Oshima, T. (1996) Molecular cloning, sequencing, and heterologous expression of a novel zinccontaining ferredoxin gene from a thermoacidophilic archeon Sulfolobus sp. strain 7. Biochem. Biophys. Res. Commun. 225, 489-493.
Williams, K., Lowe, P. N. and Leadlay, P. F. (1987) Purification and characterization of pyruvate: ferredoxin oxidoreductase from the anaerobic protozoan Trichomonas vaginalis. Biochem. J. 246, 529-536.

Zhang, Q., Iwasaki, T., Wakagi, T. and Oshima, T. (1996) 2Oxoacid:ferredoxin oxidoreductase from the thermoacidophilic archaeon, Sulfolobus sp. strain 7. J. Biochem. 120, 587-599.

Zillig, W., Stetter, K. O., Wunderl, S., Schulz, W., Priess, H. and Scholz, I. (1980) The Sulfolobus "Caldariella" group: taxonomy on the basis of the structure of DNA-dependent RNA poly-merase. Arch. Microbiol. 125, 259-269. 